\title{
General Collective Intelligence, Human-Centric Functional Modeling and the Current Limitations on Collective Design Cognition
}

\author{
Andy E. Williams, Nobeah Foundation, Nairobi, Kenya
}

\begin{abstract}
This paper explores the limits to the size and complexity of designs that can be created by humans. Beginning with the existence of a finite limit to the design output of any single individual, as a result, some design efforts require teams. However, anecdotally, when it comes to contributing to a single design objective, there is a limit to the size of groups, a limit to the diversity of skill sets, a limit to the diversity of design techniques and other problem-solving tools, and there is a limit to the range of objectives, at which the group can remain effective. Design processes are cognitive (reasoning or understanding) processes. And design tools are an automation of cognitive processes. Therefore design techniques and other problem-solving tools can be represented as the cognitive processes by which design problems are solved. The execution of these cognitive processes is then design cognition. Equating the term objective with the problem that has been defined, equating design techniques and other problem-solving tools with the cognitive processes by which those problems are solved, and defining the execution of these cognitive processes as design cognition, then since large complex designs must be created by teams, the limit to the capacity of groups for this cognition imposes a limit on the size and complexity of designs that can be created by humans. General Collective Intelligence has been defined as a system that organizes groups into a single collective intelligence with vastly greater general problem solving ability. Design based on General Collective Intelligence has been suggested to have the capacity to expand current limits to collective design cognition that might take certain classes of problems outside the capacity of groups to reliably define or solve. In any GCI based design process, Human-Centric Functional Modeling is a critical requirement in defining design problems as well as in creating the designs that solve those problems. This paper explores GCI based design, and the classes of problems that can't reliably be defined or solved without it.
\end{abstract}

\section{Keywords}

Human Centric Functional Modeling, HCFM, General Collective Intelligence, GCI, Design Cognition, Design Complexity

\section{Introduction}

The relationship between cognitive ability and design ability has been studied [1]-[3], as has the relationship between capacity to manage design complexity and cognitive ability[4]-[6], though many questions still remain in both areas. But rather than study design as it is being done, this paper explores what design processes can't yet be executed, and as a consequence what designs can't yet be created. A General Collective Intelligence or GCI is defined here as a system that organizes a group into a single collective intelligence with general problem solving ability, and with the potential for vastly greater general problem solving ability than any individual in the group. By contrast, a conventional Collective Intelligence or CI uses the intelligence of crowds to gain increased ability to solve specific problems, or a specific range of problems. While the impact of groups and collective intelligence on design has been explored [7]-[12], the impact of GCI on design has not.

If cognitive capacity places a limit on the size and complexity of designs that can be created, and if as suggested General Collective Intelligence expands the limit on a group's collective cognitive capacity to be far higher than the limit on the cognitive capacity possible for any individual, and expands the limit on the collective cognitive capacity of the group to be far higher than that of any group without GCI [14], then since GCI has not yet been implemented in the design process, there must be certain 
classes of design problems outside the capacity of groups to reliably define, and certain classes of solutions too complex to reliably be created to solve those problems. The question is how to intuitively understand what those limits to problem solving and design complexity are.

GCI is intended to mimic the complexity achieved by systems in nature. It's intuitively obvious that the 40 billion or so cells (neurons) in the brain as a natural system are capable of achieving far more complex patterns of cooperation for any purpose, including cognition, than any collection of 40 billion single-celled organisms. It's not just a matter of the equipment each cell has. It's easy to envision that even if every single-celled organism was given all the connectivity structures required to communicate with other cells, as well as all the additional structures required to perform every other function of cells in the brain, the group of single-celled organisms wouldn't suddenly start to "think". Because what would still be missing is the assignment of a specific function to a specific neuron in a specific hierarchy of functional structures. That is, what would be missing is the system of organization through which the neurons interact.

The theory of General Collective Intelligence suggests that the same principles used to organize interactions between cells in order to reliably maximize outcomes for the organism as a collection of cells, can be applied to organizing interactions between individual humans to create the capacity to maximize collective outcomes for the group. This applies to orchestrating interactions in groups so groups can collectively "reason", and applies to achieving any specific collective reasoning process in teams such as design. It also applies to scaling cooperation in order to scale that collective reasoning ability. The question is how does the lack of this collective reasoning ability define the limits to the ability of groups to achieve design complexity?

Regarding the interaction between cells in an organism, it's possible to intuitively identify the classes of things that the complex cooperation between cells in an organism should be able to do that groups of single-celled animals would not be able to do, though it might not be easy to quantify the level of difficulty of collective tasks that uncoordinated individual entities might not have the capacity to achieve. What an organism reliably can do that groups of individual cells can't includes any task for which the competition between individual interests and group interests creates a barrier to cooperation, and for which the value of cooperation must be scalable to the point at which cooperation can reliably be incentivized, and therefore can reliably occur.

By analogy with the additional level of complexity that can be achieved in interactions between cells in an organism, it's also possible to intuitively identify the classes of group design problems or group design solutions that require conceiving complex interactions between functions, where those interactions can be observed to be too complex to reliably be conceived by a group. This includes designing any process for which the number of simultaneous interactions that must occur in order to achieve an outcome creates a barrier. For such problems the capacity to detect the functions in a high enough number of simultaneous interactions must be scalable to the point at which such barriers of complexity can reliably be surpassed, and therefore sufficiently complex design to solve the problem through reconfiguration of the system can reliably occur. Design problems requiring the capacity to consider such higher order can be said to be outside the capacity of that group to define. And design solutions requiring the capacity to consider such higher order interactions can be said to be outside the capacity of the group to discover.

But before identifying specific designs that are too complex to be detected even where they are better, its important to explore how in general the processes by designs achieve their functions must be differentiated as being accomplished by individual processes or by collective processes, and how those 
processes must be differentiated as resulting in individual or collective outcomes. This distinction between designs created by collective design processes and designs created by individual ones is required for such an identification of processes that are too complex to be designed without collective intelligence to be unambiguous.

\section{Collective Processes and Outcomes vs Individual Processes and Outcomes}

The general problem solving ability of a group organized by a General Collective Intelligence, according to the model of GCI discussed in this paper, is an emergent property of the system of GCI organizing that group, as opposed to being related to the intelligence of any individual group member [17]. The difference is that the intelligence of an individual is inherently aligned with their interests, whereas the intelligence of a GCI is inherently aligned with the group's interests. Here the term "individual interests" is defined objectively as any outcome that increases fitness in achieving all the individual's functions. The term "collective interests" is defined as any outcome that increases fitness in achieving all the group's functions.

The first step in determining where the capacity to create better designs requires collective intelligence, is determining what is a better design. And making this determination requires understanding the difference between what is a better design for an individual and what is a better design for a group, which requires the ability to distinguish individual and collective outcomes. One person incentivizing an entire group to execute a process aligned with that individual's interests (even if it is their interest in helping the group) is defined here as achieving an individual outcome. Assuming that group interests alone cannot drive decision-making processes without a collective decision-making system like GCI to measure group interests and to solve the problem of achieving them, then individual outcomes include designs created by any current design process not based on GCI. On the other hand, where a group of individuals cooperate through some process that converges on the interests of the group independently of the desire of any individual in the group to serve their own interests, the outcome of that process is defined here to be a collective outcome. Collective outcomes include designs created by any GCI based design process.

There is also the question of outcome versus impact of that outcome on fitness to achieve all functions (what we intuitively recognize as well-being). An individual process of design might achieve a high magnitude of a particular outcome, but that outcome might harm the individual's well-being in the short or long-term. To compare processes like design processes, and to compare outputs of those processes like actual designs, one must therefore define the context of that comparison, including whether individual or collective outcomes achieved by those designs are being compared, and whether specific outcomes are targeted, or whether individual or collective well-being is being targeted. As an example, an individual design outcome that might be targeted is maximizing planned obsolescence. Without GCI to orchestrate cooperation across the entire value chain, sustainable designs might not be economically viable. In this situation, a design that competes to accumulate capital by increasing consumption as unsustainably as possible is the only economically viable option. The best design must then rely on this strategy of prioritizing individual economic outcomes to capture as much value from the "sinking ship" as possible, in order to have the resources to be able to develop a strategy to survive in another way if and when global tipping points are exceeded.

This highlights a paradox. While GCI based collaborative design might be better at achieving collective well-being, not all outcomes targeted by design are consistent with improving well-being. And if comparing which design processes is better, cooperation in general is simply worse at achieving certain outcomes. As an example in nature, if a cell's targeted outcome is becoming cancerous, the body's cooperation will not be as effective in cooperating to create cancer than that cell will be on its own. A 
GCI based process (one that maximizes collective outcomes) might not do as well at such individual tasks. In the case of targeting outcomes not consistent with collective well-being, GCI might create the capacity to create designs that better serve collective well-being, where doing so requires more complexity than an individual is reliably capable of, but from this perspective those are not better designs.

If GCI maximizes collective outcomes then most individuals will achieve greater outcomes through the use of GCI based design but some might achieve worse outcomes than would be the case if individual outcomes were targeted. But if an individual targets their own well-being as opposed to some outcome not directly related to their well-being, then if a GCI is defined as having the capacity to scale cooperation to the point that the value in the cooperation can reliably incentivize all participants to cooperate, then a GCI based design or other process should have the capacity to reliably increase that individual well-being as opposed to an individual design process.

In the model of cognition on which GCI is based, outcomes are selected according to impact on fitness in achieving all outcomes, which is a functional representation of well-being of the cognitive system. The FMF in which this model of the cognitive system was defined represents both individual cognition and GCI as selecting outcomes according to impact on well-being, therefore the difference between outcomes and impact of those outcomes is important. However, in addition, individuals vary widely in their knowledge of what impacts well-being. In this cognitive model, the general accuracy in the individual's understanding of what impacts well-being, and therefore the individual's ability to reliably achieve and maintain well-being, is referred to here as degree of "enlightenment". In the FMF this term has the objective and well-defined meaning as the capacity to navigate a larger volume "fitness space" of the cognitive system per unit time, so that navigating to greater fitness is reliably achievable.

Defining cognitive enlightenment as the capacity to navigate the conceptual space to reliably achieve well-being, then the individual or collective's degree of enlightenment is also related to the individual or collective's assessment of what design is better. The FMF defines all concepts as belonging to a "conceptual space" and defines all design and other cognitive processes as navigating between those concepts (those points in conceptual space). A greater degree of enlightenment implies a greater understanding of how a larger range of things (concepts in conceptual space) are related to the entity's well-being. And an understanding of how a larger range of things are related to the entity's well-being is in effect expanding the entity's consciousness of what the boundaries to itself actually are.

In this sense, the degree of enlightenment represents the degree to which the individual has expanded their consciousness of what the boundaries to their individual self actually are, so a greater impact on individual well-being is possible. As an example, if an individual only sees a full stomach as wellbeing, then they miss out on designs that do anything else, which is virtually every design. If an individual only sees their personal condition as being related to their well-being, then they miss out on designs that give them the gift of being of service to others. When the sense of self has been enlarged, then the individual will look for impact on well-being rather than on a specific outcome. And when the understanding of what impacts well-being is enlarged, the individual will look for impact on well-being for others rather than for this individual physical body alone. 
Limit to Design Outcomes Achievable by Individuals with Differing Levels of Enlightenment

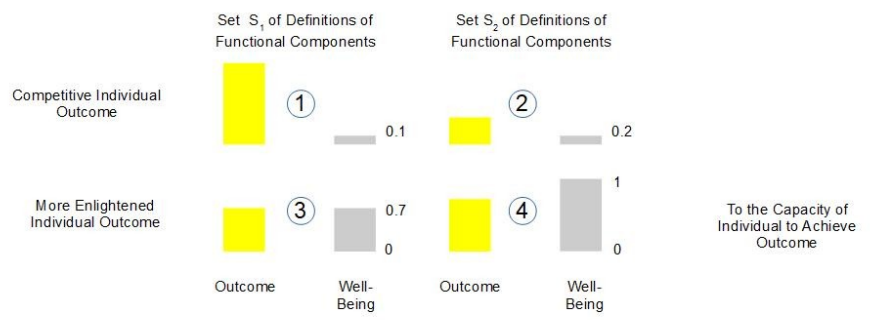

Figure 1: Defining degree of enlightenment as degree of ability to achieve well-being, an individual that focuses on using design to create a greater magnitude of any outcome in relation to that achieved by someone else (a competition based individual outcome), might have a higher capacity to achieve that outcome through design, but a lower capacity to achieve well-being through design. In gaining the ability to reliably detect a better design, it's necessary to understand not only high magnitude of a targeted outcome resulting from a design (case 1), but also a high impact on well-being resulting from another design (case 4).

In addition to increasing capacity to impact outcomes when targeting collective rather than individual outcomes, a GCI might further increase collective outcomes through an equivalent "enlightenment".

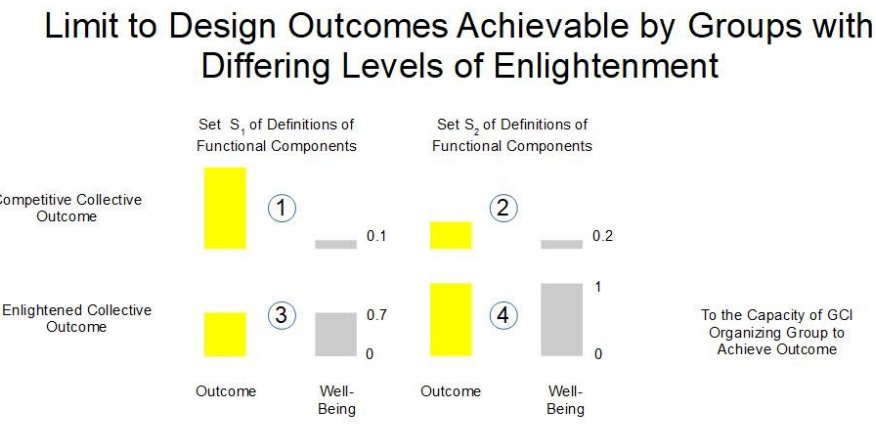

Figure 2: The ability of a group to create better designs not only depends on the collective cognitive capacity, and therefore depends on GCI, but also depends on the degree of enlightenment of the group, so the group is able to distinguish that a design resulting in high well-being (case 4) is better than a design resulting in a high outcome (case 1).

\section{Using GCI to Scale Cooperation}

GCI increases the value of impact on collective outcomes to the point that impact on collective outcomes is reliably achievable, in part because that value can be increased to the point that cooperation to achieve that outcome can reliably be incentivized. Given any process, we can scale the rate and magnitude of that process and its outcomes through execution of activities in parallel. And we can transform the outcome of that process through other processes executed in series, so that it's 
possible to achieve larger categories of outcomes. We can also execute all these parallelization and serialization processes according to the principles of collectively intelligent cooperation that are implemented by a GCI, which enable cooperation to reliably converge on collective rather than individual outcomes. By executing a cooperative process with a GCI, we can use the GCI to scale any collective outcomes of such a process.

Increasing Value of Collective Outcomes

Value of Outcome

Centralized (Individual)

Process
Proced

Collectively Intelligent

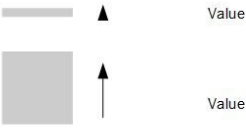

Figure 3: A centralized process is an individual process in that it can be aligned with any individual interests. Such a process might create large outcomes, but the value of outcomes possible through collectively intelligent cooperation is proposed to be higher. In design, collectively intelligent cooperation can create levels of value not possible through current centrally owned design processes. In other words, I can pay you all to do something for me. But cooperation can pay you much more to do something for yourselves. And if the collective intelligence is enlightened what you can do for yourselves is even greater.

\section{Open and Closed Design Problems}

In understanding how the capacity for collective design cognition can significantly improve design's, its also important to understand how GCI applies to different design problems. One category of design problems are those that are closed in that they target a specific outcome.

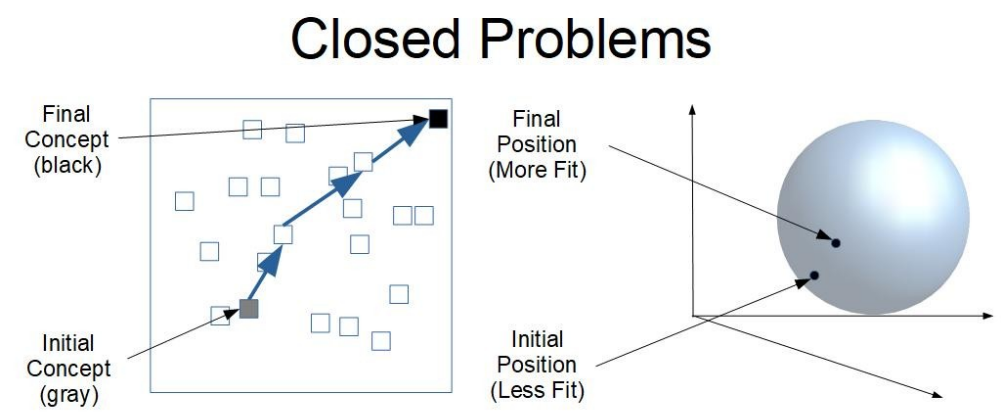

Figure 4: Closed problems can be defined in terms of any path from one concept (one point in conceptual space) to another concept, where that navigation results in changing a particular aspect of well-being.

Another are design problems that are open in targeting only an impact on some aspect of well-being. 


\section{Open Problems with High Openness}

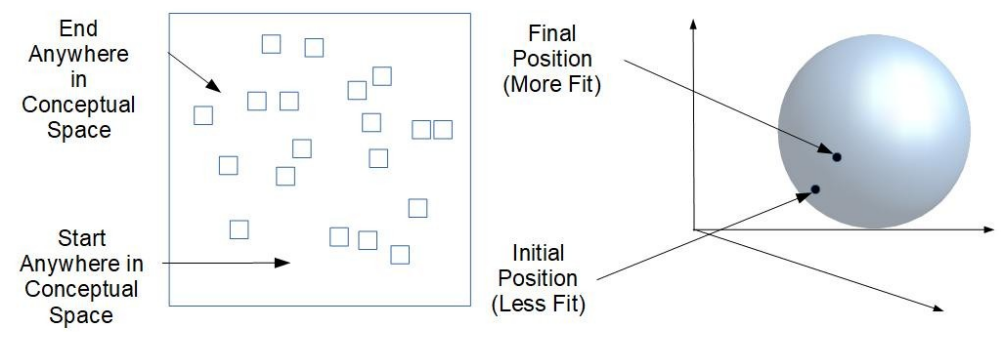

Figure 5: Problems with high openness can potentially be defined in terms of any path from any one concept (one point in conceptual space) to any other concept. In effect, they are only defined by a change in a particular aspect of well-being.

Another are design problems are moderately open in being restricted to certain outcomes. For example, given the problem of designing a "better" cutlery solution, the best solution from the perspective of this being a closed problem might be cutlery that is more easily cleaned, or easier for different sized hands to use, etc. The best solution from the perspective of this being a completely open design problem might be a menu of fresh healthy vegetables and simple meals that can be eaten with the hands, as well as providing education about why eating consciously means not working on the computer, using one's phone, or doing any thing else while eating that might prevent one from eating with one's hands due to the desire to avoid soiling that equipment.

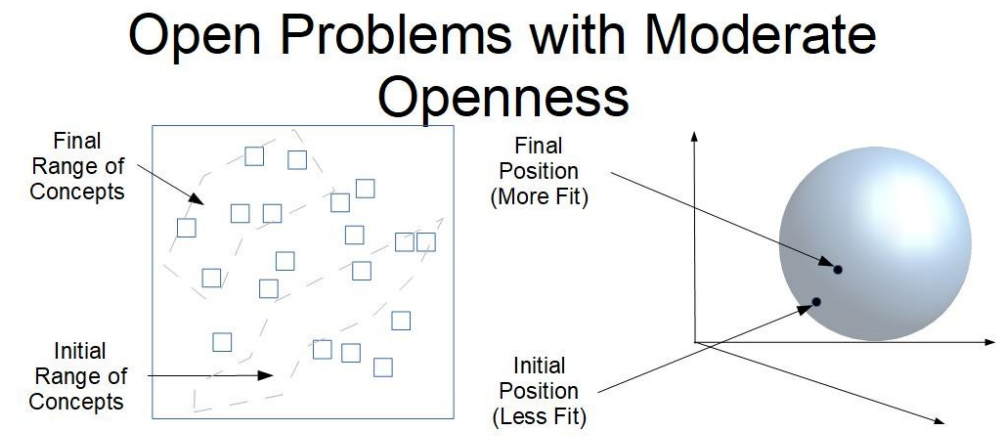

Figure 6: Problems with moderate openness can potentially be defined in terms of any path from any one in a set of concepts (one region in conceptual space) to any other in another set of concepts, where that navigation results in changing a particular aspect of well-being.

\section{Design Problems and Solutions in Conceptual Space}

From the perspective of Human-Centric Functional Modeling, design cognition processes are processes by which one generalizes problems or solutions into classes of problems or solutions that define outcomes at higher levels of abstraction. At the highest level of abstraction a problem becomes a strategic problem that targets choosing a category of outcome. The solution chooses that category. At the lowest level of abstraction, a problem becomes the problem of achieving a specific outcome. The solution achieves that outcome. Every design process becomes a process that can be added to a library that the GCI can use to increase its design problem solving ability.

GCI based design processes have been described as having the capacity to traverse a larger problem space and a larger solution space, what does this look like? Any design process used by any individual 
to achieve any design outcome can be added to a library that a GCI can make the group more intelligent in using. Any fact known by any individual can potentially be added to a library of concepts. That library of concepts and processes might be harvested from observation alone, of the individual's outputs, and the sequence of those outputs. Or the individual might provide those concepts and processes themselves.

\section{Design Complexity}
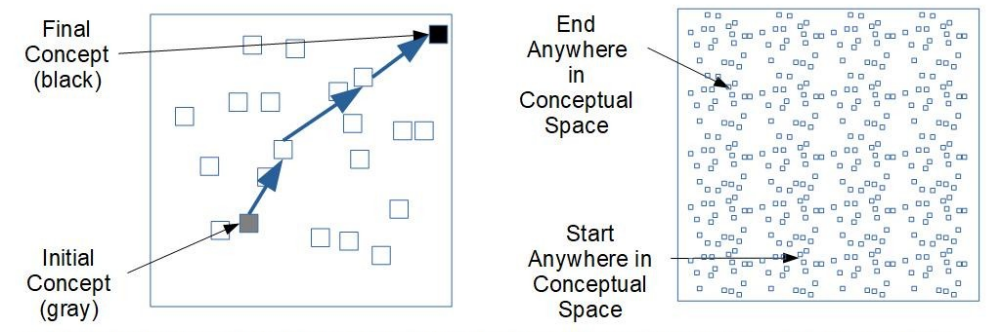

Figure 7: Problems with low design complexity (left)

navigate simpler paths between concepts resolved at lower resolution (larger). Problems with high design complexity (right) navigate more complex paths between concepts resolved at higher resolution (smaller), and potentially do so at a much higher level of abstraction so they might start and end anywhere in conceptual space. Without GCI to create the required cognitive capacity to conceive such problem definitions or their solutions, such solutions might not even be recognized as solving the same problem, much less be recognized as being far better solutions.

\section{GCI as Biomimicry of Nature's Design Processes}

General Collective Intelligence mimics the adaptive problem-solving methodology nature uses to enable a system to achieve stability of "fitness" in response to any problem posed by its environment. Nature is represented as using this methodology to expand the limitations to scaling or centralizing cooperation as needed to reliably solve any "fitness" problem that exists in the cooperation domain. And nature is represented as using this methodology to expand the limitations to scaling the complexity of any interaction in the cognitive system that is required by whatever reasoning process needs to be executed to reliably solve any "fitness" problem that exists in the reasoning domain. When applied to human groups, General Collective Intelligence removes the barriers to collective reasoning being able to discover more complex interactions where those barriers must be overcome in order to even conceive of the existence of certain collective problems or to conceive of their solutions. Without it, the lack of significant ability to collectively reason, and the lack of ability to scale cooperation through collective reasoning processes, are hidden bugs in our decision-making, and as a result of these bugs, we are constrained to using the wrong solutions to solve the wrong problems.

Nature is also represented as designing systems through such adaptive problem-solving processes. In design, General Collective Intelligence copies nature's ability to reliably create and execute the design processes required to better solve collective challenges, even where they are too complex to be created through current human design processes. 
Current human design processes are not yet reliably capable of many outcomes nature can reliably accomplish, for example, nature is reliably capable of designing structures such as the human brain itself, or the complex proteins that give spider's silk its remarkable tensile strength, or any other of the great many complex designs nature has originated. The GCI hypothesis suggests that products or services that are sufficiently complex to require designs with what it defines as Nth order interactions between functions in those designs, where $\mathrm{N}$ is higher than individuals are capable of, or higher than groups are capable of without GCI, cannot reliably be created without GCI. Nature's adaptive problem solving process is an example of a problem solving process capable of conceiving problems and solutions involving much higher order interactions than the human mind is reliably capable of. Nature is capable of designs that are too complex to be reliably achieved through current human design processes, that is, which involve Nth order interactions with $\mathrm{N}$ high enough that those interactions are not reliably discoverable through current design processes. GCI based design processes can be demonstrated to have the capacity to significantly increase $\mathrm{N}$, and therefore to sufficiently increase human capacity for design complexity.

Increasing the complexity of structures that can be reliably designed is one purpose of General Collective Intelligence. Another is facilitating the complex cooperation required to make those products or services sustainably viable. Current human cooperation is not yet reliably capable of creating ecosystems that make manufacturing any product or service sustainably viable in the USA. Design does not exist in a vacuum. Current human design processes are unstable in the sense that they are driven by a very limited set of very changeable factors, and so are no longer viable when those factors change. Nature on the other hand does not design any of its products or services in isolation, but creates entire ecosystems within which that design is sustainably viable.

\section{The Characteristics of GCI Based Design Process Execution}

The ability to scale cooperation implies a GCI must remove the barriers to any individual participating in the design process. A GCI must facilitate any information provided by any individual becoming part of a data store the group can use to improve it's problem solving ability, and must facilitate every process defined by any individual becoming part of a library the GCI uses to increase the group's ability to solve problems. In design, a GCI then requires the capacity to reliably converge on providing the best available information, to the best available individuals, to execute the best available design processes in order to maximize design outcomes.

This requires breaking up the design process and designs themselves into a minimal set of functional components which any design process or design can be represented in terms of and be compared, as well as requiring a methodology for comparing the fitness of any design process or design in achieving its targeted outcome. And it requires other functionality listed in table 1 that characterizes GCI based design process execution:

\section{Component of Model}

Functional modeling (problems and solutions)

Functional decomposition

Functional domain bridging Different domains in which different functions are more fit in achieving the same purpose are identified. These domains are bridged by using a set of weights which identify the best function in each domain.

Functional fitness

\section{Description}

Components are modeled only by function to remove prejudice for or against any given implementation. Functional components are decomposed into their most basic functional Every functional component is assigned some projected and actual fitness in achieving its function. 
Functional stability

Functional adaptation
For functional components to persist they must display some degree of stability in fitness to function.

For functional components to persist in a changing environment they must have the ability to adapt their function. One of the most important domains of adaptation is cooperation. Functional components must interact according to the principles of decentralized cooperation in order to maximize outcomes for each. Functions must be prioritized according to the principles of centralized cooperation in order to maximize outcomes globally.

Table 1: Components of GCI process execution.

\section{Human-Centric Functional Modeling of Design Processes for Execution by a GCI}

In this approach, conceptual entities (any entity that can be conceived) are separated into two categories; static entities that don't change state, and dynamic entities that do. Static entities are described in terms of their functions, identified by the outcomes the concepts can be observed as functioning to achieve. Dynamic entities are also described in terms of function, but instead the minimal set of observed functions by which they change state, and from which all other observed functions can be composed. This process of observation is human-centric because it is limited to observations that can be made within innate human perception rather than being in terms of constructs such as beliefs or intellectual theories about the mechanisms by which those functions are implemented, or in any other terms that can't yet be validated within observation.

Dynamic entities (systems) are not only described in terms of the minimal set of human-observable functions capable of describing all their behaviors, but in addition are described in terms of the states accessible through these functions. The set of these states forms a "functional state space" through which the system moves. In addition, the system being operational (alive in the case of biological organisms) is represented as the system being confined to a bounded region of a "fitness space" that represents its fitness to execute all its functions.

One set of minimal functional components that might be used to break up the design process into a set of common components any design process can be represented in terms of and be compared is below: 


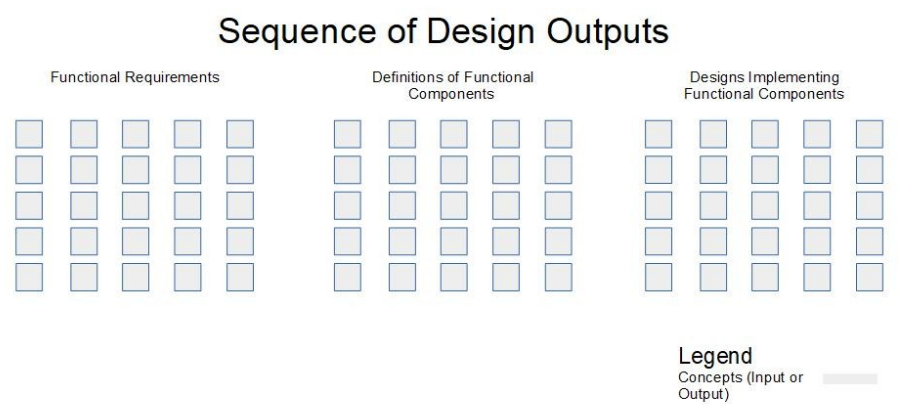

Figure 8: The first process in any design methodology defines functional requirements (left) specifying what the design must do. The next process in design (center) defines the functional components that functionality must be split across. The last process design (right) specifies implementations for the functional components. These outputs are represented as sets of concepts in a conceptual space.

The process of GCI based design must create the ability to use cooperation to methodically vary all the properties of each process so that exploring the entire conceivable design process space is more reliably achievable. This replicates nature in that nature has demonstrated the capacity to explore a very large space of potential processes, to come up with the complex processes we observe in living organisms.

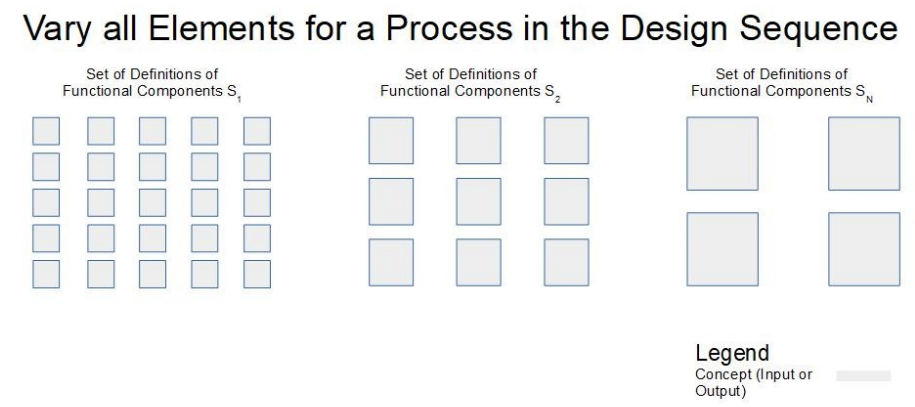

Figure 9: The second process in design defines the functional components that the design functionality must be split across. Gaining the ability to navigate more of the possible design configuration space (i.e. the ability to create a wider range of designs) requires the ability to methodically explore all design possibilities, such as the impact of using one set of functional component definitions S1 (left), or the impact of using any other set SN (right) that objectively varies in some property, such as the number of components defined.

One set of minimal functional components that might be used to break up any design process into a set of common components that any design can be represented and be compared in terms of is below: 


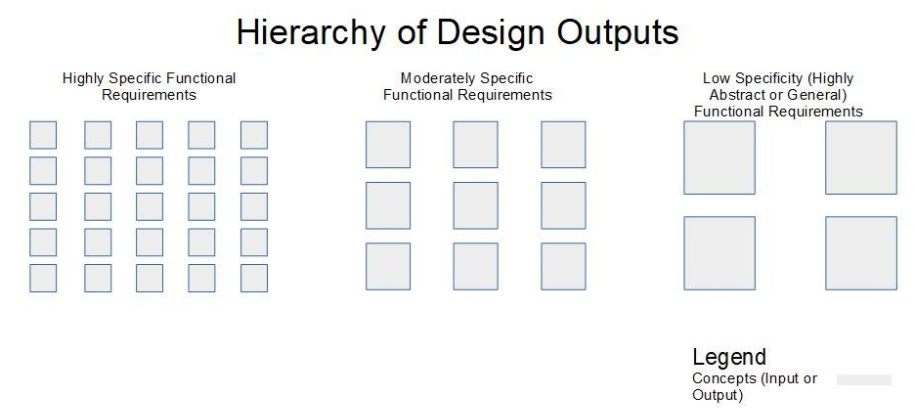

Figure 10: Design outputs can also be defined in a hierarchy of abstraction from highly specific (left) to very general (right).

All design inputs or outputs can in turn be related through these categories of design functions.

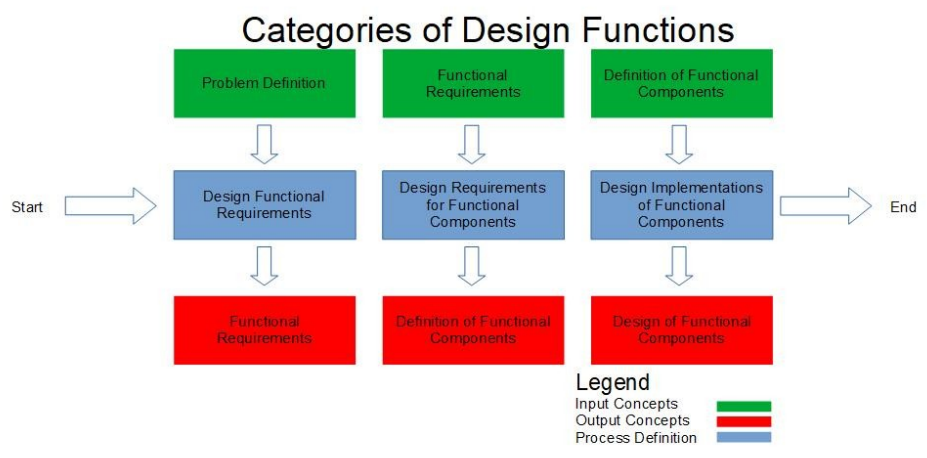

Figure 11: Each process in the sequence of any design methodology requires inputs and produces outputs.

A proposed GCI research program (the Large Scale international COllaborative Research or LSCORE initiative) has been defined to elaborate that functional modeling approach and to define the models of products, services, and design processes to be used in the approach. This research will also elaborate the GCI algorithm used to orchestrate these collective design processes so these projects are possible.

\section{Example: The Design for Change Nairobi Challenge}

By splitting the GCI functionality over such a number of projects and by implementing such projects in an intelligent sequence, it may be possible to implement it in phases that reduce the cost per project to the point that implementation becomes reliably achievable. A proposed Collective Intelligence based Program to Accelerate Achievement of the Sustainable Development Goals (CIPAA-SDGs) aims to do just that. Where phase I relies only on the Social Impact Marketplace platform, phase II relies on GCI platforms in healthcare, renewable energy, education and other areas. Phase III defines a Collaborative Design platform in order to facilitate a project aiming to leverage massive design collaboration with the capacity to accommodate millions of participants. This project, called "Design for Change Nairobi" aims to use this massive collaboration to lower the cost of designing modular smart, sustainable housing solutions, that incorporate collectively intelligent cooperation with renewable energy, healthcare, or other solutions, to reliably increase income and decrease costs for people in the slums of Nairobi by a sufficient amount per month to move those people out of poverty. The principles required to implement collectively intelligent design have been explored elsewhere [15]. 
In addition, because GCI removes the barriers to scaling cooperation, where the value of that cooperation is positive, that value can then be increased to the point that this cooperation is reliably self-funding. Using this self-funding property, the first three parts of this ten phase program aim to achieve the capacity to reliably drive $\$ 10$ billion USD of private funding towards these goals.

The proposed GCI based design program (the Design for Change Nairobi Challenge) requires a library of functional models of products and services and design processes that can be used by a GCI to significantly increase the group's ability to solve design problems. Full implementation of GCI implies the ability to enable any user to leverage any design process and any available information in order to

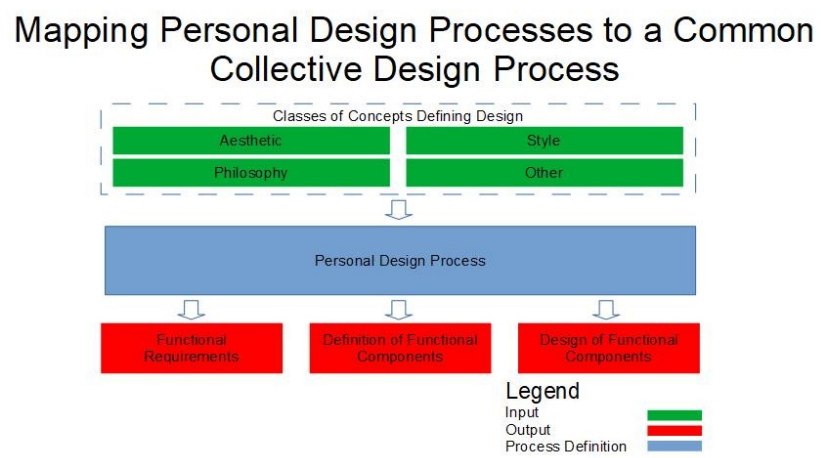

Figure 12: Any personal approach to design, whether incorporating a given aesthetic, a style, or any other attribute, can be mapped to the same functional components of all design methodologies. Mapping to a common collective design process enables contributions from an unlimited number of designers to converge on a single design.

In order to have the capacity for general problem solving in design this requires the ability to navigate to any design process from any relationship to any design concept. This might be comprised of a wizard that watches what you're designing, asks you what it is, then suggests the best approaches that you have the skills to use. A great many suggestion processes might exist. The GCI would need to use whichever one maximizes the designer's fitness at achieving whatever is eventually revealed to be the targeted outcome.

But if a GCI can use any available process to collectively define the problem and the solution, and will continually measure outcomes and refine its idea of which solution is most fit, then how can we limit a GCI to design? Taking the CIPAA-SDGs as an example, define a set of collaborative design processes. Expand that set in phases. For example:

$\begin{aligned} & \text { Expanding GCI Design Functionality Over the Versions of the Collaborative } \\ & \text { Design Platform }\end{aligned}$
$\begin{array}{ll}\text { Version of the Collaborative Design } & \text { Functionality } \\ \text { Platform } & \text { Design Implementations of Specific } \\ \text { Version } 1 & \text { Components } \\ \text { Version } 2 & \text { Design Component Definitions } \\ \text { Version } 3 & \text { Design Functional Requirements }\end{array}$


Version 4

Make Customized Contributions to

Design Process

Table 2: Potential implementation of GCI based design functionality over the planned versions of a Collaborative Design Platform.

This is the same way that we limit our thoughts to a given problem and potential solutions.

Classes of Design Problems and Solutions Requiring General Collective Intelligence

What classes of processes require Nth order cooperation in order to be reliably achievable? What subset of those are processes to design a product or service? What classes do those products or services fall into? What are the features of the problems those products or services solve?

\begin{tabular}{|l|l|}
\hline Classes of Design Problems and Solutions Requiring General Collective Intelligence \\
\hline Feature of Design Problem or Solution & $\begin{array}{l}\text { Nth Order Cooperation or Other Nth Order } \\
\text { Interaction Required }\end{array}$ \\
\hline $\begin{array}{l}\text { Sustainability (Sustainable machines that must } \\
\text { share a limited number of components across } \\
\text { enough other designs that maximizing value by } \\
\text { minimizing consumption is viable.) }\end{array}$ & $\begin{array}{l}\text { Coordinate the design of N products, where N is } \\
\text { increased to the point that sustainability (rather than } \\
\text { planned obsolescence) is economically viable [13]. }\end{array}$ \\
\hline $\begin{array}{l}\text { Self-Containment in Biological Machinery } \\
\text { (Biological machines that must produce some } \\
\text { functionality from higher order interactions } \\
\text { between the limited set of components they } \\
\text { contain, this includes self-reproduction, or self- } \\
\text { healing.) }\end{array}$ & $\begin{array}{l}\text { Search for designs that achieve targeted outcomes } \\
\text { through combinations of N interactions between } \\
\text { functional components, where N is increased to the } \\
\text { point that functionality can be reliably designed } \\
\text { even if too complex for individual cognition. }\end{array}$ \\
\hline
\end{tabular}

Table 3: Classes of design problems not reliably conceivable, and classes of design solutions not reliably solvable without GCI.

Assume a cognitive system (in this case a collective one) has the capacity to scale cooperation to execute reasoning processes to whatever order $\mathrm{N}$ is required to execute processes within that system. Assuming that Nth order cooperation requires GCI in order to be reliably achievable when $\mathrm{N}$ is sufficiently high, then any process requiring the capacity to scale cooperation to the Nth order then requires GCI to be reliably achievable.

\section{Conclusion}

This paper has explored the concept of design cognition from the point of view of a model of cognition defined through a Functional Modeling Framework based on Human Centric Functional Modeling. And it has identified a number of design problems whose solutions might be too complex to be reliably achievable with current design processes that do not leverage a model of General Collective Intelligence to increase capacity for collective design cognition. In order to validate the existence of this limit to complexity, and the importance of overcoming it, more work is required to define functional models of design processes, to assess the complexity of those models in terms of the "Nth order interactions" defined in this paper, and to assess whether limits to $\mathrm{N}$ are actually observed.

Finally, a system of "General Collective Intelligence" needs to be distinguished from a system providing more generic "intelligence". Currently, software is the only feasible way to implement a GCI. So GCI is essentially software. Once such software is implemented, and once functional models 
of designs have been defined for use within design processes for which functional models have also been defined, then a GCI might enable a lone designer to incorporate the work of a great many others, and by themselves do the work that entire teams of designers are required for today. Once no orchestration of any further collective human activity is required to achieve such productivity it might not be immediately clear why this is a type of "collective intelligence" rather than simply software that increases "design intelligence". The answer is that all the functional models of every component of every product or service, and all the functional models of every process, must be defined and input in order to be available. While creating a complex design using such a system might just seem to require one person in this case, it actually took an entire civilization to create the GCI that made it possible.

\section{References}

[1] Sajjad Nazidizaji, Ana Tomé, Francisco Regateiro, Does the smartest designer design better? Effect of intelligence quotient on students' design skills in architectural design studio, Frontiers of Architectural Research, Volume 4, Issue 4, 2015, Pages 318-329, ISSN 2095-2635, https://doi.org/10.1016/j.foar.2015.08.002.

[2] Love, T: 2001, Concepts and affects in computational and cognitive models of designing, in JS Gero and ML Maher (eds), Computational and Cognitive Models of Creative Design, University of Sydney, Sydney, pp. 3-23.

[3] Shah, J., Smith, S. M. , and Woodward, J., 2009, "Development of Standardized Tests for Design Skills," International Conference on Engineering Design (ICED09), Stanford, CA.

[4] J.S Busby, Error and distributed cognition in design, Design Studies, Volume 22, Issue 3, 2001, Pages 233-254, ISSN 0142-694X, https://doi.org/10.1016/S0142-694X(00)00028-4.

[5] Zafer Bilda, John S. Gero,

The impact of working memory limitations on the design process during conceptualization, Design

Studies, Volume 28, Issue 4, 2007, Pages 343-367, ISSN 0142-694X, https://doi.org/10.1016/j.destud.2007.02.005.

[6] Shah, Jami; Woodward, Jay; Smith, Steve, Development of Standardized Tests for Design Skills

DS 58-10: Proceedings of ICED 09, the 17th International Conference on Engineering Design, Vol. 10, Design Education and Lifelong Learning (2009), Page(s): 269-280

[7] Maher M.L., Paulini M., Murty P. (2011) Scaling Up: From Individual Design to Collaborative Design to Collective Design. In: Gero J.S. (eds) Design Computing and Cognition '10. Springer, Dordrecht

[8] Mercedes Paulini, Paul Murty \& Mary Lou Maher (2013) Design processes in collective innovation communities: a study of communication, CoDesign, 9:2, 90-112, DOI: 10.1080/15710882.2012.716850 [9] Murty, P., Mercedes, P., \& Maher, M. Lou. (2010). Collective Intelligence and Design thinking. DTRS8 Interpreting Design Thinking, 309-315.

[10] Paulini M, Murty P, Maher ML, Understanding collective design communication in open innovation communities, Journal of CoCreation in Design and Arts 9:90-112

[11] Paulini, M. \& Maher, M.L. \& Murty, P.. (2011). The role of collective intelligence in design: A protocol study of online design communication. 687-696.

[12] Benjamin G. Shaw (2010) A cognitive account of collective emergence in design, CoDesign, 6:4, 225-243, DOI: 10.1080/15710882.2010.533184

[13] Williams, Andy E. "General Collective Intelligence as the Emerging Paradigm in Human-Centric Design for Sustainability", accepted for publication in the proceedings of the peer-reviewed $26^{\text {th }}$

International Sustainable Development Research Society Conference that will take place the 15-17th of July, 2020 in Budapest, Hungary

[14] Williams, Andy E. “A Model for General Collective Intelligence.” AfricArXiv, 30 Apr. 2020. Web. 
[15] Williams, Andy E. "The Collective Intelligence Based Program to Accelerate Achievement of the Sustainable Development Goals as a Case Study for Collectively Intelligent Program Design." SocArXiv, 30 Apr. 2020. Web.

[16] Williams, Andy E. "Use of Human-centric Functional Modeling to Maximize Convergence in Integrative Research.” AfricArXiv, 15 May 2020. Web.

[17] The Relationship Between Collective Intelligence and One Model of General Collective Intelligence, Andy E. Williams, Computational Collective Intelligence, 11th International Conference, ICCCI 2019, Hendaye, France, September 4-6, 2019, Proceedings, Part II, Pages 589-600 